\title{
Reversal-Specific Learning Impairments After a Binge Regimen of Methamphetamine in Rats: Possible Involvement of Striatal Dopamine
}

\author{
Alicia Izquierdo*,', Annabelle M Belcher², Lori Scott', Victor A Cazares', Jack Chen', Steven J O'Dell', \\ Melissa Malvaez ${ }^{2}$, Tiffany $\mathbf{W u}^{2}$ and John F Marshall ${ }^{2}$ \\ 'Department of Psychology, California State University, Los Angeles, CA, USA; ${ }^{2}$ Department of Neurobiology and Behavior, University of \\ California, Irvine, CA, USA
}

\begin{abstract}
A growing body of evidence indicates that protracted use of methamphetamine (mAMPH) causes long-term impairments in cognitive function in humans. Aside from the widely reported problems with attention, mAMPH users exhibit learning and memory deficits, particularly on tasks requiring response control. Although binge mAMPH administration to animals results in cognitive deficits, few studies have attempted to test behavioral flexibility in animals after mAMPH exposure. The aim of this study was to evaluate whether mAMPH would produce impairments in two tasks assessing flexible responding in rats: a touchscreen-based discrimination-reversal learning task and an attentional set shift task (ASST) based on a hallmark test of executive function in humans, the Wisconsin Card Sort. We treated male Long-Evans rats with a regimen of four injections of $2 \mathrm{mg} / \mathrm{kg} \mathrm{mAMPH}$ (or vehicle) within a single day, a dosing regimen shown earlier to produce object recognition impairments. We then tested them on (I) reversal learning after pretreatment discrimination learning or (2) the ASST. Early reversal learning accuracy was impaired in MAMPH-treated rats. MAMPH pretreatment also selectively impaired reversal performance during ASST testing, leaving set-shifting performance intact. Postmortem analysis of [ ${ }^{125} \mid$ I]RTI-55 binding revealed small (10-20\%) but significant reductions in striatal dopamine transporters produced by this mAMPH regimen. Together, these results lend new information to the growing field documenting impaired cognition after mAMPH exposure, and constitute a rat model of the widely reported decision-making deficits resulting from $\mathrm{mAMPH}$ abuse seen in humans.

Neuropsychopharmacology (2010) 35, 505-5I4; doi:I0. I038/npp.2009. I55; published online 30 September 2009
\end{abstract}

Keywords: reversal, attentional set shifting; behavioral flexibility; inhibitory control; psychostimulants; compulsivity

\section{INTRODUCTION}

Methamphetamine (mAMPH) is a highly addictive psychostimulant drug that can result in impaired cognition in humans (Ornstein et al, 2000; Simon et al, 2000; Bechara et al, 2001; Volkow et al, 2001c). Research on human users suggests that the cognitive deficits extend beyond learning and memory, into the realm of inhibitory control and executive function (Rogers et al, 1999; Ornstein et al, 2000; Simon et al, 2000; Bechara et al, 2001; Kalechstein et al, 2003; Nordahl et al, 2003; McCann et al, 2008). The use of animal experimental models can help elucidate the neurobiological mechanisms by which abuse of this drug brings about such cognitive sequelae. Many researchers

\footnotetext{
*Correspondence: Dr A Izquierdo, Department of Psychology, California State University, Los Angeles, 515I State University Drive, Los Angeles, CA 90032, USA, Tel: + I 323343 2283, Fax: + I 323 343 228I, E-mail: aizquie@calstatela.edu

Received 21 April 2009; revised 17 August 2009; accepted 21 August 2009
}

have used animal models of mAMPH exposure that incorporate short, moderate-to-high dose schedules of mAMPH administration, intended to mimic human abusers' bingeing patterns of mAMPH use. In addition to uncovering brain mechanisms, studies of drug-exposed animals help address the complicated question of whether cognitive impairments are a direct consequence of the drug, secondary to other conditions arising from the drug, or representative of preexisting vulnerabilities in the population of drug users - vulnerabilities that predispose them to becoming addicts (Verdejo-Garcia et al, 2008). Administering acute, binge doses of mAMPH to rats produces impairments in several domains of learning and memory (Bisagno et al, 2002; Schroder et al, 2003; Belcher et al, 2005; $\mathrm{He}$ et al, 2006; Herring et al, 2008a) and often without evidence of neurotoxicity (Belcher et al, 2006; Nagai et al, 2007).

At sufficiently high doses, binge mAMPH exposure causes dose-dependent damage to the dopamine (DA) system, injury that has been reported in both nonhuman primates and rodents (Ricaurte et al, 1982; Gibb et al, 1987; O'Dell 
et al, 1993; Villemagne et al, 1998), with the ventral region of caudate-putamen (CP) being particularly vulnerable to mAMPH's neurotoxic effects (Eisch et al, 1992). Converging evidence suggests that human mAMPH abusers also have reductions in brain DA indices (Eisch et al, 1992; Wilson et al, 1996; Volkow et al, 2001b; Volkow et al, 2001a; Volkow et al, 2001c).

Compulsive drug-seeking behavior bears a striking resemblance to the disinhibited behavior after damage to the frontal cortex and is thought to be due, in part, to the many plastic responses that occur in the brain after repeated psychostimulant use (Everitt and Robbins, 2005; Dalley et al, 2008; Everitt et al, 2008). In fact, changes in the striatum may be at the root of such compulsive behavior (Everitt and Wolf, 2002), with measures of enhanced DA activity in dorsal striatum associated with higher selfreported levels of craving and strength of psychostimulant habit in humans (Volkow et al, 2006) and increasing 'habitization' of behavior in rats (Takahashi et al, 2007). In addition, lesions of the medial or ventral striatum produce perseverative, compulsive responding on reversal learning in monkeys (Clarke et al, 2008) and strategy set shifting in rats (Block et al, 2007), both assays for different forms of behavioral flexibility. These effects are similar to those well documented after lesions to the ventromedial prefrontal cortex, namely the orbitofrontal cortex (OFC; Schoenbaum and Shaham, 2008).

Given the foregoing, there appears to be an association between the neurobiological consequences of heavy psychostimulant use and compromised inhibitory control mechanisms. To date, animal studies of mAMPH effects on cognition have focused on testing for impairments using traditional learning and memory paradigms. Surprisingly, with few exceptions (Dalley et al, 2007; Daberkow et al, 2008) there has been relatively little development in an animal behavioral model investigating the long-term effects of mAMPH on behavioral flexibility. To our knowledge, there have been no investigations of discrimination reversal learning and attentional set shifting after binge-type administrations of mAMPH. The aim of this study was to investigate the effects of binge mAMPH exposure on two hallmark measures of flexible behavior in rats: visual discrimination reversal learning and attentional set shifting. We chose a treatment regimen of four injections of $2 \mathrm{mg} / \mathrm{kg}$ mAMPH because this regimen results in (1) acute hyperthermia during treatment, (2) a posttreatment object recognition impairment, and (3) significant but moderate $(\sim 20 \%)$ depletions of DA transporter in ventral CP while sparing the serotonin transporter in the areas examined (hippocampus, perirhinal cortex) (Belcher et al, 2008). This dose is also well tolerated by the exposed animals. As widespread neurotoxicity appears not to result from treatment with this moderate dose, we focused our quantification of DAT to the striatum, the area in the brain most sensitive to the DA-depleting effects of mAMPH (Eisch et al, 1992).

\section{MATERIALS AND METHODS}

\section{Subjects}

Thirty-seven adult male Long-Evans hooded rats (275-300 g at start of experiment) were obtained from Charles River Laboratories (Raleigh, NC) and individually housed, with water ad libitum, under a standard $12 \mathrm{~h}$-light $/ 12 \mathrm{~h}$-dark cycle (lights on $0700-1900 \mathrm{~h}$ ) at a temperature of $22^{\circ} \mathrm{C}$. Rats were food restricted to $85 \%$ free-feeding body weight for behavioral testing (see Experiments 1 and 2). Protocols for this research were approved by the Institutional Animal Care and Use Committees of the University of California, Irvine and California State University, Los Angeles. Acquisition, maintenance, handling, procedures, and care of the animals were in accord with the NIH Guide for the Care and Use of Laboratory Animals.

\section{Drug Treatments}

On the day of mAMPH injections, rats were kept in large, clear Plexiglas cages measuring $40 \mathrm{~cm}$ (length) $\times 40 \mathrm{~cm}$ (width) $\times 38 \mathrm{~cm}$ (height) in groups of no more than seven animals each. Ambient room temperature was kept at $23 \pm 1.5^{\circ} \mathrm{C}$. Rats were given injections of d-mAMPH (Sigma, St Louis, MO; $2 \mathrm{mg}$ free base $/ \mathrm{kg}$, sc) or physiological saline solution (SAL; $1 \mathrm{ml} / \mathrm{kg}, \mathrm{sc}$ ) at 2 -h intervals for a total of four injections. This dose was chosen to minimize motor impairments and to circumvent any stress response produced by an interaction with food restriction, given the known deleterious effects of stress on behavioral flexibility (Holmes and Wellman, 2008). Animal body temperatures were monitored $60 \mathrm{~min}$ after each injection: by rectal probe (Experiment 1) or by temperature transponders (IPTT-300; BioMedic Data Systems, Seaford, DE) implanted subcutaneously along the dorsum between the animals' scapula the day before drug treatments (Experiment 2); Table 1.

Table I Body Temperatures of Rats Used in Experiment I (Visual Discrimination Reversal) and Experiment 2 (Attentional Set Shift Task)

Time 0 I h after first injection I h after second injection I h after third injection I $\mathrm{h}$ after fourth injection

\begin{tabular}{|c|c|c|c|c|c|}
\hline Experiment I saline & & $38.5 \pm 0.1$ & $37.6 \pm 0.3$ & $36.6 \pm 1.4$ & $37.0 \pm 1.8$ \\
\hline Experiment I mAMPH & & $38.9 \pm 0.3$ & $38.5 \pm 0.4$ & $38.8 \pm 0.2$ & $39.5 \pm 0.4$ \\
\hline Experiment $2 \mathrm{mAMPH}$ & $36.9 \pm 0.4$ & $39.5 \pm 0.3$ & $38.8 \pm 0.5$ & $39.2 \pm 0.4$ & $39.0 \pm 0.5$ \\
\hline
\end{tabular}

Temperatures $\left({ }^{\circ} \mathrm{C}\right)$ were recorded $\mathrm{I} h$ after each injection as well as before the injection regimen ('Time 0', for Experiment 2 only). 


\section{Experiment 1. Visual Discrimination and Reversal Learning}

Rats used in this experiment were acclimated to the testing room for $15 \mathrm{~min}$ and fed immediately on returning to the homecage. All behavioral testing took place between $0800 \mathrm{~h}$ and $1600 \mathrm{~h} \mathrm{5-6}$ days per week. As outlined in the steps below, rats were shaped, pretrained to nosepoke a touchsensitive screen, and trained on a discrimination problem (eg, stimulus pair) all before treatment with drug. They were then treated with mAMPH or SAL, given a posttreatment 'retention test' and administered a reversal of reward contingency.

Apparatus. Operant chambers (\#80004, Lafayette Instrument Co., Lafayette, IN), measuring $35.6 \mathrm{~cm}$ (length) $\times 27.9 \mathrm{~cm}$ (width) $\times 33.7 \mathrm{~cm}$ (height) were each housed within a soundand light-attenuating cubicle (\#83018DDP Lafayette Instrument Co., Lafayette, IN). Each operant chamber was outfitted with a touch-sensitive, 12" LCD flat screen (EloTouch, Menlo Park, CA). The chamber floor was covered with a clear Plexiglas sheet to facilitate mobility. The touchscreen and a single houselight were located at one end of the chamber; a tone generator, a pellet receptacle and a pellet dispenser, at the other end. The pellet dispenser delivered $45 \mathrm{mg}$ dustless sucrose pellets (BioServ, Frenchtown, NJ). Stimulus presentation, reward delivery and contingencies were controlled by custom-designed software developed for use in nonhuman primate experiments (Ryklin Software, Inc). The equiluminant stimuli were the same as those reported in an earlier study (Izquierdo et al, 2006b).

\section{Behavioral testing.}

Handling and accommodation to food rewards: One week on arrival to the vivarium, each rat was handled for a minimum of $10 \mathrm{~min}$ once per day for 5 days before behavioral testing. During this time, animals were given a small amount of $45 \mathrm{mg}$ sucrose pellets ( $\sim 20$ pellets) in their homecage after each day of handling to accustom them to the food rewards.

Food restriction: Food restriction to no more than $85 \%$ free-feeding body weight began during the handling period and when rats weighed a minimum of $275 \mathrm{~g}$ (between 8 and 10 weeks of age). Rats were weighed three times per week to ensure a healthy body weight throughout testing.

Acclimation, shaping, and pretraining: During acclimation, rats were required to eat pellets out of the pellet tray before exposure to any stimuli on the touchscreen. Shaping then involved the concurrent disappearance of a stimulus presented on the touchscreen and a 'reward event': illumination of a houselight in the sound-attenuating cubicle, illumination of the pellet tray light, onset of 2-s auditory tone, and provision of a $45-\mathrm{mg}$ sucrose pellet. At any point during shaping, rats could be rewarded for a 'nosepoke' on the touchscreen by this 'reward event.' Criterion for shaping occurred when rats ate 60 sucrose pellets within 30 min.

Pretraining involved three cumulative stages: (1) touchrats must touch the stimulus on the touchscreen by nosepoking the stimulus; (2) initiate-rats must initiate the onset of the next trial by nosepoking the pellet receptacle door; (3) punish - rats get 'punished' by a (house)light-out, the absence of the pellet receptacle light, and the absence of the auditory tone that usually signals reward. Instead, the trial is 'timed out,' rendering rats unable to initiate the next trial for $5 \mathrm{~s}$. Criterion for each phase of pretraining was 60 completed trials (touches) and no pellets remaining in the pellet receptacle in $30 \mathrm{~min}$.

Visual discrimination learning: Rats were presented with two two-dimensional, equiluminant white stimuli on a black background and trained according to predetermined reinforcement contingencies: Stimulus $A$ resulted in a food reward $(\mathrm{A}+)$, whereas nosepoking the other stimulus, $\mathrm{B}$, resulted in a $5 \mathrm{~s}$ timed-out punishment $(\mathrm{B}-)$. Designation of the rewarded stimulus was counterbalanced across treatment groups. The custom software enabled stimuli to be presented on the screen indefinitely until the animal nosepoked one of the stimuli. Only small preprogrammed 'response windows' overlying the stimuli were sensitive to nosepoking: nosepoking outside of the response window was undetected; nosepoking within it was either correct or incorrect, depending on reward contingency. Left/right presentation of the $S+$ was pseudorandom, according to a Gellerman schedule generated by Ryklin Software Inc. There were 60 total trials per session (and one session per day) with a 10-s ITI. For this learning phase, rats were required to reach a criterion of $85 \%$ correct out of 60 trials across each of 2 consecutive days. Performance was assessed according to three measures: session percent correct, session perseveration index (P.I., measured by dividing the number of consecutive errors in a row before switching response by the total number of errors within a session), and the number of sessions to reach performance criterion of $85 \%$ correct across two sessions.

All rats were given a 'reminder' session the day before treatment to account for differences in the recency of exposure to the reward contingencies in discrimination learning.

Posttreatment retention and reversal learning: After treatment with $\mathrm{mAMPH}$, rats were given 3-5 days of rest (eg, no behavioral testing, in homecage), followed by a test for retention of the discrimination problem. Rats were then required to respond to a reversal in reward contingency: nosepoking the previously incorrect stimulus was now rewarded by provision of a sucrose pellet. Thus, testing on reversal learning began 5-8 days post-mAMPH or SAL treatment. As in the earlier phase, criterion was set at a mean score of $85 \%$ correct out of 60 trials across two consecutive sessions. Performance was assessed according to the same three measures described above.

\section{Experiment 2. Attentional Set Shift Task}

Apparatus. A separate group of male, Long-Evans rats weighing $275-300 \mathrm{~g}$ at the time of $\mathrm{mAMPH}$ treatments was used. Attentional set shift task (ASST) procedures were conducted according to Birrell and Brown (2000). Rats were trained in a Plexiglas arena that measured $36.8 \mathrm{~cm}$ (height) $\times 45.7 \mathrm{~cm}$ (width) $\times 68.6 \mathrm{~cm}$ (length). The box was divided equally into thirds so that each compartment was 
$22.9 \mathrm{~cm}$ long. The front of the apparatus was further divided into two separate sections where the bowls were contained separately, to avoid animals having access to both bowls simultaneously. In addition, access to each compartment (and bowl) at the front of the box could be restricted by an opaque, removable divider. The back third of the apparatus (inter-trial chamber) was separated from the other compartments with a removable divider, and rats were placed in that chamber at the beginning of each trial. Access to the inter-trial chamber was blocked once a trial was in progress. Food rewards (one-half of a Frosted Cheerio, General Mills, Golden Valley, MN) were buried half-way down ceramic bowls $(3.8 \mathrm{~cm}$ tall having an internal diameter of $8.3 \mathrm{~cm})$. Rats were trained on successive days to make discriminations based on two dimensions: media of varying textures (eg, vermiculite, confetti, gravel), or scents (eg, paprika, thyme or oregano). Scents could be mixed interchangeably with media so that combinations of the two dimensions were possible, but pairs of scents or media were kept constant (eg, cumin was always presented with cinnamon; vermiculite was always presented with gravel).

\section{Behavioral testing.}

Food restriction: Rats were given 3-5 days of rest (eg, no behavioral testing, in homecage) after mAMPH or SAL injections before food restriction began, allowing for weight stabilization before food restriction. During food restriction, rats were fed $15 \mathrm{~g}$ of food per day, and weight was monitored daily to maintain a target weight of $85 \%$ normal weight. To habituate the animals to the feeding bowls, and to familiarize them with digging for rewards, food was given in small ceramic bowls in the home-cage for several days before training. ASST training began on the 14th day of food deprivation. Rats were returned to free feeding once testing was complete, and they were kept on food deprivation for no more than 30 days.

Habituation: After the 14-day food-deprivation (17-19 days after $\mathrm{MAMPH}$ or SAL injections), rats were habituated to the arena for $5 \mathrm{~min}$ before training. To familiarize the animals with digging for a food reward, animals were presented with two baited unscented bowls filled with home cage bedding. Once the animal had retrieved the food reward, the animal was moved back to the inter-trial chamber and the bowls were re-baited. The divider was then lifted, allowing the animal access to the bowls. All subsequent trials were conducted in this same manner. Once the animal was reliably digging for a food reward, training on the task was begun.

Training: During the training phase, rats were trained on two simple discriminations (SDs), one scent-based (thyme $v s$ paprika), and the second media-based (vermiculite $v s$ plastic beads). All rats were trained on the same discriminations, in the same order. Criterion completion of training was achieved when the rat made a correct discrimination for six consecutive trials. Most rats completed this training during a single day. Rats that did not learn to dig for food reward within the day were returned to their home-cage and a second attempt at training was made 1-2 days later. A maximum of three attempts were made to train each rat, with overall success in $>90 \%$ of the animals.
Testing paradigm: The day after rats had completed training on the two SD training trials, testing began. A trial was initiated by raising the dividers, giving the rat access to the two bowls, only one of which was baited. For each phase, the rat was given four discovery trials, whereby the rat was allowed to dig in both bowls (even though only one was baited) to retrieve the food reward. Errors were recorded during the discovery trials, but did not count toward trials to criterion (Birrell and Brown, 2000). On subsequent trials, if the rat dug in the unbaited (incorrect) bowl, an error was recorded, and the trial was terminated. In a single-test session, rats were given the following discrimination phases to learn: (1) SD, a scent-based discrimination (nutmeg* vs cloves); (2) compound discrimination (CD), media (paper squares $v s$ shredded paper) dimension is introduced, scent is still rewarded, irrespective of medium (nutmeg*/paper squares and nutmeg*/shredded paper); (3) CD-reversal (CDr), previously unrewarded scent is now rewarded, irrespective of medium (cloves ${ }^{*} /$ paper squares and cloves $* /$ shredded paper); (4) intradimensional shift (ID), animal must still attend to scent and correctly discriminate the rewarded scent, but novel scents (cinnamon $^{*} v s$ cumin) and media (foam triangles $v s$ straws) are introduced; (5) ID-reversal (IDr), previously unrewarded scent is now rewarded, irrespective of medium (cumin ${ }^{\star}$ I foam triangles and cumin $*$ straws); (6) extradimensional shift (ED), rat is trained to attend to medium cues ( $\frac{1}{4}$ foam shells $^{*} v s$ crushed foam) and ignore scent cues (celery seed vs sumac); (7) ED-reversal (EDr), previously unrewarded medium is now rewarded, irrespective of scent (crushed foam ${ }^{\star} /$ celery seed and crushed foam ${ }^{\star} /$ sumac). During each phase, the rats were tested until they had achieved a criterion of six consecutive correct choices. Two measures were used to quantify performance in each phase: (1) trialsto-criterion (the number of trials taken to reach criterion), and (2) the number of errors made in reaching criterion. The order of the discriminations and the exemplar pairings were always the same, but the pairs of exemplars were counterbalanced between groups. Typically, rats completed all phases of testing within a single day.

\section{Experiment 3. $\left[{ }^{125} \mathrm{I}\right] \mathrm{RTI}-55$ Binding to DA Transporters}

A separate group of male, Long-Evans rats weighing 275-300 $\mathrm{g}$ at the time of treatment was given binge $\mathrm{mAMPH}$ $(4 \times 2 \mathrm{mg} / \mathrm{kg}$, s.c., $N=6)$ or saline $(0.9 \%$ sterile saline, s.c., $N=3$ ) injections as described above and was killed 1 week later. The reason for the use of a separate group of rats was to afford an accurate estimate of DAT binding at a time point that corresponded with commencement of testing in Experiment 1. Rats were anesthetized with an overdose of sodium pentobarbital $(250 \mathrm{mg} / \mathrm{kg}$, i.p.), decapitated, and their brains removed and frozen at $-20^{\circ} \mathrm{C}$ by immersion in isopentane. A measure of $20 \mu \mathrm{m}$-thick coronal sections were cut on a cryostat at the level of the anterior striatum (AP coordinates $+1.7 \mathrm{~mm}$ to $+0.8 \mathrm{~mm}$, according to Paxinos and Watson, 2003), thaw-mounted on Vectabond-treated glass slides and stored at $-20^{\circ} \mathrm{C}$ until used for autoradiography. Warmed slides removed from the $-20^{\circ} \mathrm{C}$ freezer were preincubated in a solution of assay buffer $(10 \mathrm{mM}$ $\mathrm{NaPO}_{4}, 120 \mathrm{mM} \mathrm{NaCl}, 100 \mathrm{mM}$ sucrose) containing $100 \mathrm{nM}$ fluoxetine for $5 \mathrm{~min}$ to remove endogenous ligands that 
could interfere with subsequent radioligand binding. As $\left[{ }^{125}\right.$ I]RTI-55 binds with high affinity to both DAT and SERT, $100 \mathrm{nM}$ fluoxetine was included in both the preincubation and incubation media to block radioligand binding to SERT (Boja et al, 1992). After preincubation, the sections were incubated in a solution of assay buffer containing $25 \mathrm{pM}\left[{ }^{125} \mathrm{I}\right] \mathrm{RTI}-55$ and $100 \mathrm{nM}$ fluoxetine for $2 \mathrm{~h}$. The sections were then rinsed twice for $2 \mathrm{~min}$ each at $4^{\circ} \mathrm{C}$ in assay buffer, then once for $10 \mathrm{~s}$ in $4^{\circ} \mathrm{C}$ distilled water. The rinsed slides were then rapidly dried under a stream of heated air. The dried slides and $\left[{ }^{14} \mathrm{C}\right]$-containing autoradiographic standards were apposed to Hyperfilm MP (GE Healthcare) for $48 \mathrm{~h}$ before development.

Quantification of ${ }^{125}$ I]RTI-55 binding was done using an MCID image analyzer (InterFocus Imaging; Cambridge, England). Image densities were converted to [ ${ }^{125}$ I]RTI-55 binding levels using a calibration curve based on images of the standard slides packed with each film. Regional densities of RTI binding were obtained by outlining the desired structures on their respective $\left[{ }^{125} \mathrm{I}\right] \mathrm{RTI}-55$ images. Values obtained represented the average of measurements taken from both hemispheres in a total of four sections per animal. For analysis, the image of striatum was first subdivided into CP and nucleus accumbens septi (NAc). The CP was then subdivided into dorsal (dCP) and ventral (vCP) parts, which were separately quantified for [ $\left.{ }^{125} \mathrm{I}\right] \mathrm{RTI}-$ 55 binding (see Figure 4).

Statistics. Temperature data were analyzed using repeatedmeasures ANOVA. Visual discrimination reversal learning data (percent correct and P.I.) were analyzed with repeatedmeasures ANOVA for early (sessions 1-3), middle (sessions 4-6), and late (sessions 7-9) learning phases. Retention of visual discrimination and sessions to criterion were assessed using independent-samples $t$-tests. ASST behavioral data (trials to criteria, errors) and $\left[{ }^{125} \mathrm{I}\right] \mathrm{RTI}-55$ binding data were assessed using repeated measures ANOVA. $t$-Tests were used to compare performance of $\mathrm{mAMPH}$ and SAL groups during each phase of the ASST. Within-group comparisons between performance in the ED and ID phases were performed by paired $t$-tests. In all instances, two-tailed tests were used, with $p$-values equal to and $<0.05$ considered statistically significant and trends noted at $p$-values less than and equal to 0.10 . Data are presented as mean \pm SEM values and were analyzed with SPSS.

\section{RESULTS}

\section{Experiment 1}

Effect of $M A M P H$ on visual discrimination: pretreatment learning and posttreatment retention. Rats learned the visual discrimination problem in an average of 3.2 sessions ( \pm 1.4 SEM). Stimulus-reward assignment (either A $+B-$ or $\mathrm{A}-\mathrm{B}+)$ did not influence the rate of acquisition, thus data were collapsed for all subsequent analyses.

One day before treatment with $\mathrm{mAMPH}$ or SAL, rats were given a 'reminder' session of the discrimination problem. They were then tested for their retention of the initial visual discrimination problem after treatment. As shown in Figure 1, the mAMPH and SAL groups scored similarly on

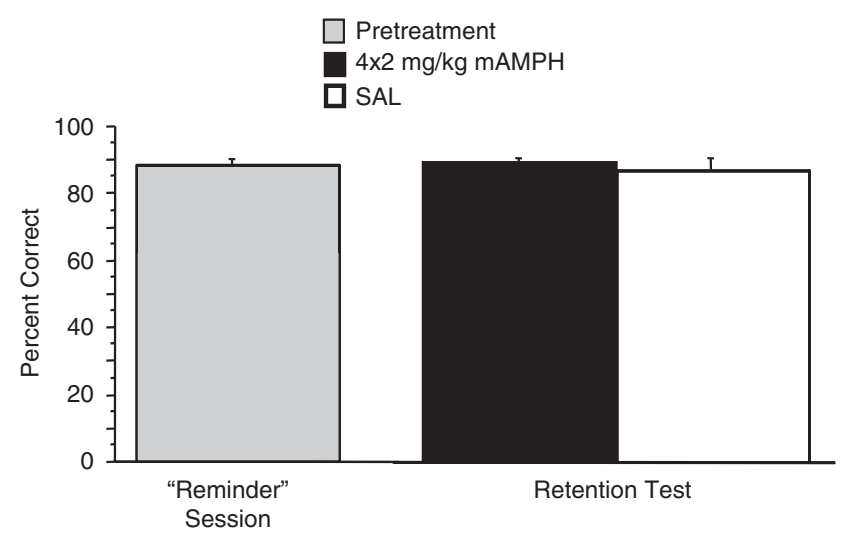

Figure I Retention of a pretreatment visual discrimination problem. Rats were given a 'reminder session' where they were exposed to the discrimination problem just learned. Three days after treatment with $\mathrm{mAMPH}$ or $\mathrm{SAL}$, they were given a retention test. There were no significant differences between the $\mathrm{mAMPH}$ - and SAL-treated groups $(n=7 \mathrm{mAMPH}, n=5 \mathrm{SAL})$. Data are means $\pm \mathrm{SEM}$.

this memory test (percent correct: $\mathrm{mAMPH}, 89.97 \pm 1.26$; SAL, $87.98 \pm 3.62 ; \mathrm{t}(10)=0.59, p=0.57)$.

Effect of $M A M P H$ on reversal learning. Consistent with the known acute effects of this $\mathrm{mAMPH}$ dose $(4 \times 2 \mathrm{mg} / \mathrm{kg}$, s.c. $)$, the $\mathrm{mAMPH}$-treated rats showed a trend toward elevated body temperatures during the treatment regimen (Table 1; $\left.\mathrm{F}_{1,9}=3.41, p=0.06\right)$.

Although there were no significant treatment differences on overall sessions to criterion (additional details below), we explored early reversal learning for two reasons: (1) initial reversal learning taps into greater inhibitory control and constitutes the phase in which the greatest perseveration is seen (Jones and Mishkin, 1972) and (2) dopaminergic manipulations selectively affect early reversal learning using touchscreen-response methodology in rodents (Izquierdo et al, 2006b). Thus, repeated-measures ANOVA were used to assess treatment differences on each phase of learning: early (sessions 1-3), middle (sessions 4-6), and late (sessions 7-9) reversal learning. As learning rates differed across animals (eg, two mAMPH-pretreated rats and one SAL-pretreated rat lacked nine full sessions), rats' two-session criterion average of $85 \%$ or better was carried forward to complete late phase reversal learning. There was a significant effect of treatment in early reversal performance such that the mAMPH-pretreated group was significantly impaired relative to the control group (percent correct: $\mathrm{mAMPH}, 29.4 \pm 4.2 ; \mathrm{SAL}, 44.0 \pm 4.5 ; \mathrm{F}_{1,10}=4.6$, $p=0.05$; data shown in Figure 2). There was no significant treatment $\times$ session interaction in early, middle, or late phases of learning, but significant effects of session were observed: all rats showed evidence of improvement with increasing session number (all F-values $>2.0$, all $p$-values $<0.05)$. Importantly, there were no differences between treatment groups on middle or late reversal learning. There were also no differences in Perseveration Index between treatment groups during early, middle, or late phases. Stimulus-reward assignment (either $\mathrm{A}+\mathrm{B}-$ or $\mathrm{A}-\mathrm{B}+$ ) did not explain the treatment difference in percent correct scored during early reversal, nor was there a significant 


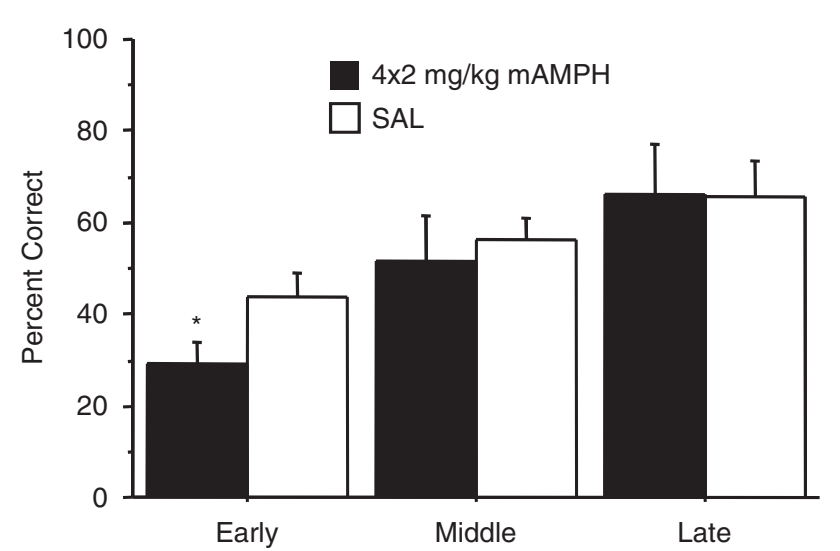

Figure 2 Early discrimination reversal learning differs between $\mathrm{mAMPH}$ and SAL-treated groups. Performance on reversal learning was subdivided into early (sessions I-3), middle (sessions 4-6), and late (session 7-9) phases. MAMPH-pretreated rats scored lower percent correct on early reversal phase only $(n=7 \mathrm{mAMPH}, n=5 \mathrm{SAL})$. Bars represent group means \pm SEM $* P=0.05$ vs SAL.

interaction of stimulus-reward assignment by session. As stated earlier, overall rate of learning on reversal of reward contingencies was not different between treatment groups (sessions to criterion: $\mathrm{mAMPH}, 11.6 \pm 2.8$; SAL, $12.2 \pm 2.4$; $\mathrm{t}(10)=-0.16, p=0.88)$.

\section{Experiment 2}

ASST performance. The mAMPH-treated rats $(4 \times 2 \mathrm{mg} / \mathrm{kg}$, s.c.) subsequently tested for ASST performance showed a significant elevation in body temperatures during the treatment regimen (Table 1; $\mathrm{F}_{1,8}=10.44, p<0.02$ ).

During training, the rats readily learned to discriminate between food-baited bowls based on either scents or media. Testing was conducted over seven phases, as described in Materials and Methods and Figure 3. Repeated measures ANOVA was used to investigate differences in trials-tocriteria and errors made for the mAMPH and SAL groups. For the trials-to-criterion measure, the effects of treatment (SAL vs mAMPH; $\mathrm{F}_{1,14}=5.87, p=0.03$ ) and phase $\left(\mathrm{F}_{6,84}=29.37, p<0.001\right)$ and the phase $\times$ treatment interaction $\left(\mathrm{F}_{6,34}=3.17, p=0.007\right)$ were significant. Subsequent tests revealed that the mAMPH group took significantly more trials to reach criteria than did SAL animals for the $\operatorname{IDr}\left(\mathrm{F}_{1,14}=20.5, p<0.001\right)$ and $\operatorname{EDr}\left(\mathrm{F}_{1,14}=4.94, p=0.04\right)$ phases and showed a trend toward treatment difference in the $\operatorname{CDr}$ phase $\left(\mathrm{F}_{1,14}=3.09, p=0.10\right)$. For the errors measure, the effects of treatment $\left(\mathrm{F}_{1,14}=4.51, p=0.05\right)$ and phase $\left(\mathrm{F}_{6,84}=22.5, p<0.001\right)$ were significant but the interaction was not $\left(\mathrm{F}_{6,84}=1.75, p=0.12\right)$.

In contrast to the effects of mAMPH pretreatment on reversals, no evidence of impairment in shifting of attention was found. That is, both mAMPH- and SAL-pretreated groups required more trials to reach criteria, and committed more errors, for the discrimination requiring an extradimensional shift, compared with intradimensional shift (paired sample $t$-tests, $p$-values $<0.025$ ). However, the ED-ID difference scores of the two groups did not differ significantly, suggesting that mAMPH did not influence animals' attentional set-shifting abilities.
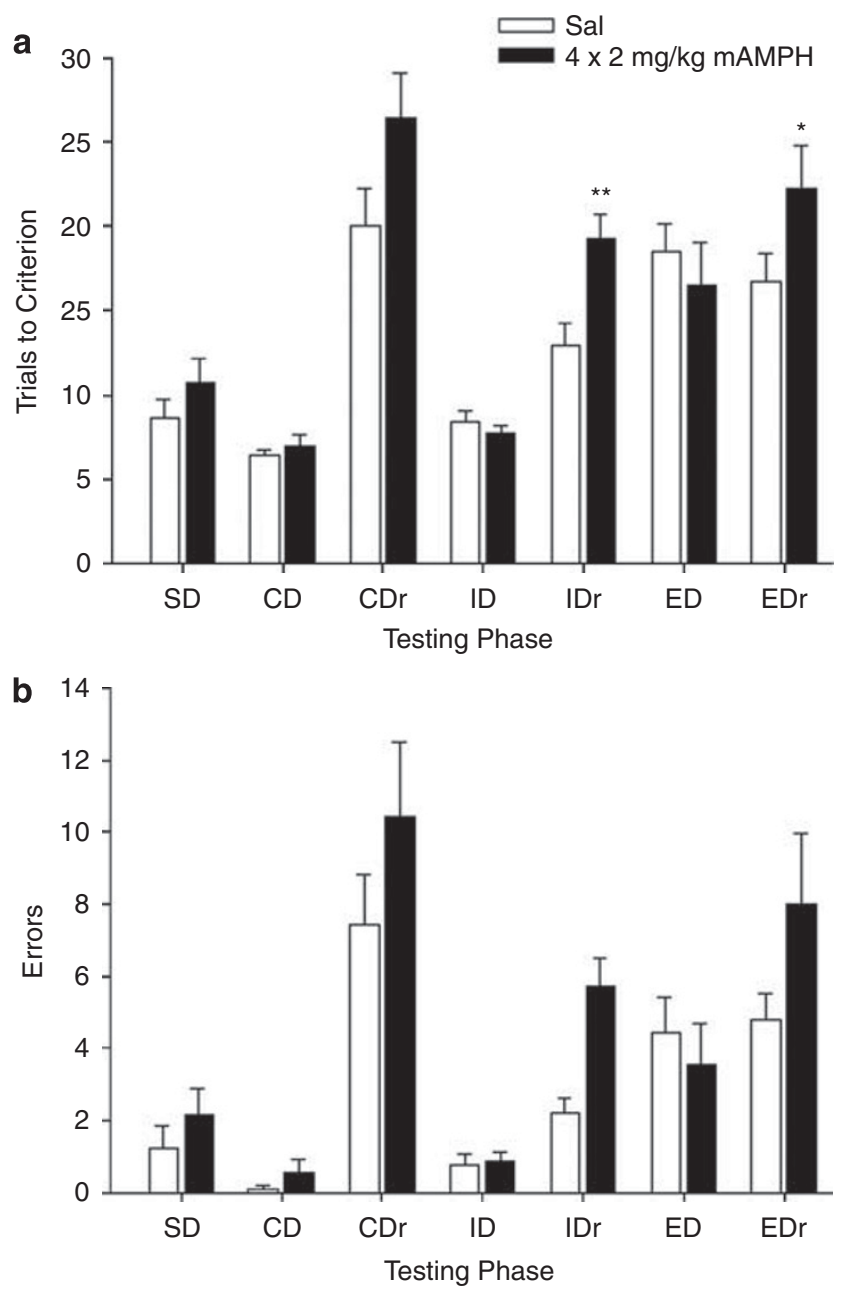

Figure 3 Reversal learning is impaired and set shifting is intact in the ASST task after mAMPH treatment. (a) mean ( \pm SEM) trials to reach criterion (six successive correct trials) for each phase of the attentional set shift paradigm. (b) mean ( \pm SEM) errors made in each phase of the ASST paradigm $(n=9 \mathrm{mAMPH}, n=7 \mathrm{SAL})$. See Materials and Methods for details. $* 0 \leqslant 0.05$; $* * * 0.001$.

\section{Experiment 3}

$\left[{ }^{125}\right.$ I]RTI-55 binding to DA and serotonin transporters. Analysis of $\left[{ }^{125} \mathrm{I}\right] \mathrm{RTI}-55$ binding revealed that treatment of rats with $4 \times 2 \mathrm{mg} / \mathrm{kg}$, s.c. mAMPH regimen produced a significant $(\sim 14 \%)$ depletion in DAT within $\mathrm{CP}$, whereas NAc DAT was unaffected. Further analysis of the CP revealed that the mAMPH-induced reductions in DAT occurred in both the dorsal and ventral subdivisions, with the greatest effect being evident in the ventral region (with depletions averaging about $18 \%$, relative to controls; Figure 4).

\section{DISCUSSION}

To our knowledge, the current report offers the first evidence of binge mAMPH-induced deficits in two forms of discrimination reversal learning, a widely used assay of flexible cognition. These results fit well with findings of human mAMPH-dependent individuals' executive dysfunction, especially when these individuals are confronted with 


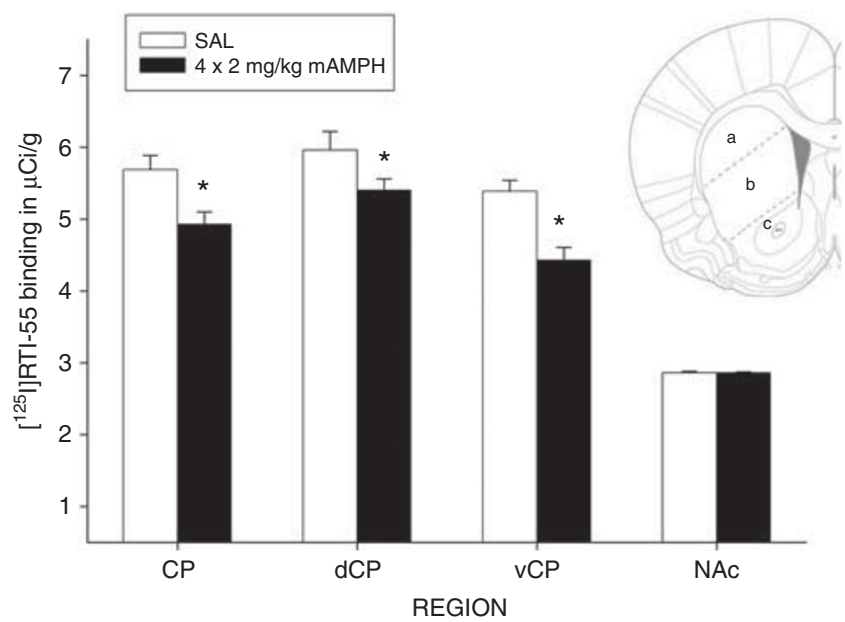

Figure $4 \quad\left[{ }^{125} \mid\right] R T I-55$ binding to striatal dopamine transporters (DAT). Mean \pm SEM values (in $\mu \mathrm{Ci} / g$ ) for DAT binding in the striatum of LongEvans rats exposed to $4 \times 2 \mathrm{mg} / \mathrm{kg}$, s.c. methamphetamine (mAMPH; $N=6$ ) or saline (SAL; $N=3$ ) dosing regimen I week earlier. Values were generated by quantitative autoradiography of ligand binding. Initial readings were taken from whole caudate-putamen (CP, areas labeled $a$ and $b$ ), followed by readings in the dorsal (dCP, labeled a) and ventral (vCP, labeled $b$ ) subdivisions of the $C P$, and in the nucleus accumbens septi (NAc, labeled c). Representative section = Bregma $+1.44 \mathrm{~mm}$. $* \mathrm{mAMPH}$ different from SAL, $p<0.02$.

changes in contingencies (Rogers et al, 1999; Kim et al, 2005; Chung et al, 2007; Han et al, 2008). This inability to update responding in favor of more adaptive choices is reminiscent of the impairments seen after damage to OFC. Altered striatal dopaminergic function and its relationship to OFC function are discussed below.

\section{Reversal-Specific Impairment After $4 \times 2 \mathrm{mg} / \mathrm{kg}$ mAMPH Treatment}

Rats treated with a single-day binge regimen of mAMPH $(4 \times 2 \mathrm{mg} / \mathrm{kg})$ were impaired on reversal learning as assessed in two tasks: visual discrimination reversal learning and attentional set shifting. The effect on the former was highly specific, as it was contained within the earliest phase of reversal learning when animals typically commit more errors in favor of the previously learned reward contingency (Jones and Mishkin, 1972; Izquierdo et al, 2006b). MAMPH-treated rats recovered from this early-phase impairment, however, reaching criterion at a comparable rate to SAL-treated rats. There were no other differences in performance that would indicate global changes in attention or motivation such as delayed approach to food reward or stimuli.

As earlier studies report object recognition memory impairments resulting from binge doses of $\mathrm{mAMPH}$, rats in this study were tested on their retention of a pretreatment discrimination problem and were found to be unimpaired. This suggests that the resulting impairment might have more to do with perturbations in behavioral flexibility and inhibitory control and less to do with deficits in learning and memory processes per se. It remains possible, however, that rats would have been impaired at learning a novel discrimination pair (not assessed), or that memory impairments would have manifested with higher treatment doses of mAMPH. Future studies should investigate effects of different doses and patterns of mAMPH administration on this task.

The reversal-specific impairment was also found in the ASST with slower overall learning (more trials to criterion) in $\mathrm{mAMPH}$-treated rats during intradimensional and extradimensional reversals, with a trend toward the same difference in the $\mathrm{CD}$ reversal phase. These results are consistent with earlier reports of a reversal impairment in rats after sensitizing regimens of either amphetamine (Fletcher et al, 2005) or cocaine (Schoenbaum et al, 2004). In addition, we found no deficits in treated rats' ability to discriminate different scents and media or to shift attention from one modality to another. This finding of intact set-shifting ability stands in contrast to findings of attentional set-shifting impairments after sensitizing regimens of amphetamine (Fletcher et al, 2005; Featherstone et al, 2008). Notably, both of these studies used a 5-week, escalating-dose amphetamine regimen that results in robust behavioral sensitization to this drug, a pattern of dosing that differs markedly from the single-day binge administration used in this study. This distinction bears mentioning since sensitizing regimens of either amphetamine or mAMPH have been shown to blunt certain forms of learning by interfering with the plasticity that would otherwise occur (Kolb et al, 2003). The possibility exists that the differences in patterns of administration (low doses of amphetamine delivered at regular intervals across several weeks $v s$ a single day, higher-dose regimen of mAMPH) elicit different results via distinct mechanisms.

Our results differ from those of Daberkow et al (2008), reporting large dopaminergic depletions $(\sim 55 \%)$ in the dorsomedial striatum of mAMPH-treated rats in the absence of a reversal learning impairment. Several methodological differences between the two studies could account for the lack of correspondence (eg, different doses of mAMPH administered, different treatment times used, use of a response-reversal rather than stimulus choice reversal task, different rat strain), yet one might expect that large dopaminergic depletions would produce similarly large impairments on a task such as motor response reversal learning, a striatal-dependent task. If, however, an imbalance of frontocortical-striatal systems underlies mAMPH effects on inhibitory control (described in more detail in the next section), then one could argue that smaller dopaminergic depletions in the striatum might lead to a greater imbalance, which in turn could lead to reversal learning impairments. The methods used here preclude establishing a causal relationship.

A potential noteworthy contributor to reversal learning impairments after mAMPH is stress. Elevated levels of corticosterone (CORT) are seen 1-72 h after mAMPH treatment (Herring et al, 2008b). Few groups have investigated stress indices such as CORT after single day, experimenter-administered mAMPH in rats, though the mechanism by which stress and mAMPH interact to bring about neurotoxicity remains an important focus of research (Szumlinski et al, 2001; Tata and Yamamoto, 2008). Earlier studies in rodents have reported behavioral flexibility impairments following brief, acute stressors as well as changes in dendritic morphology in areas of the frontal cortex (Izquierdo et al, 2006a; Holmes and Wellman, 2008). 
Future investigations should identify and control for the ancillary effects of stress on mAMPH's action.

\section{Putative Neural Mechanisms for Reversal Impairments After mAMPH Treatment}

In Experiment 3, mAMPH-induced reductions in DAT were found in both dorsal and ventral divisions of the $\mathrm{CP}$, with ventral $\mathrm{CP}$ most affected. In both magnitude and regional specificity, the pattern of reduction in striatal DAT after a single-day $4 \times 2 \mathrm{mg} / \mathrm{kg} \mathrm{mAMPH}$ dosing regimen agrees with the results of Belcher et al (2008). Such modest neurotoxicity to the ascending dopaminergic system could be sufficient to affect the intricate balance in the frontostriatal system involved in critical aspects of executive control (Robbins, 2005; Dalley et al, 2008). Compromised striatal circuitry may underlie the maladaptive responding reported in Experiment 1. This interpretation accords well with a recent report that medial striatal lesions in monkeys produce reversal learning impairments virtually indistinguishable from that brought about by OFC damage (Clarke et al, 2008; Man et al, 2008).

Recent positron emission tomography (PET) studies in humans provide evidence that striatal DA neurotransmission is important for performance in tasks of inhibitory control such as card sorting (Monchi et al, 2006). This conclusion is corroborated by evidence in animals that DA transmission in the striatum is critical for the flexible shifting of response (O'Neill and Brown, 2007; Haluk and Floresco, 2009). These more recent findings echo the longstanding theory that an intimate relationship exists between the basal ganglia network and areas like the OFC in response to changing reward contingencies (Hollerman et al, 2000). In addition, it has been reported that individual human differences in reversal learning performance reflect variations in baseline striatal DA synthesis capacity, measured by PET (Cools et al, 2009) and that different subregions of the striatum contribute to reversal learning over, for example, spatial working memory (Clatworthy et al, 2009). Importantly, low levels of D2 receptors in the striatum of mAMPH users have been associated with decreased metabolic activity in OFC (Volkow et al, 2001b; Volkow et al, 2004)

Both OFC and prelimbic cortex project to dorsomedial striatum in the rat (Berendse et al, 1992) and both frontocortical subdivisions are thought to contribute to different aspects of behavioral flexibility (Ragozzino, 2007). The pattern of behavioral impairments observed in this study-spared attentional shifting and impaired reversal learning-mimics what occurs after damage to OFC (McAlonan and Brown, 2003) but not medial frontal cortex (Birrell and Brown, 2000) damage. The evidence reviewed above supports the conclusion that the mAMPH-induced impairment of dopaminergic systems of the striatum is sufficient to impair reversal learning. However, the possibility that this binge mAMPH regimen had lasting, direct influence on cortex merits consideration. Effects of higher doses of mAMPH on integrity of neurons in somatosensory cortex (Commins and Seiden, 1986; Eisch and Marshall, 1998) have been demonstrated. However, at the doses used here, single-day binge mAMPH administration produces no loss of serotonin transporter in the cortical areas sampled (Belcher et al, 2008), while even higher binge mAMPH doses - sufficient to produce $>50 \%$ reductions in striatal DA - result in no significant depletion of frontal cortex DA (Ohmori et al, 1993). Several groups have reported that a single-day binge mAMPH administration leads to altered functioning of cerebral cortex, affecting metabolism and immediate early gene expression of several cortical areas (including OFC; Pontieri et al, 1990; Belcher et al, 2009). Elsewhere, we have hypothesized that these functional changes in cerebral cortex of binge mAMPHtreated rats occur secondarily to the decreases in striatal DA, achieved through alterations in striato-nigro-thalamocortical loops (Marshall et al, 2007; Belcher et al, 2009). The basal ganglia-cortical loop hypothesis also provides a useful framework for interpreting the above-mentioned reports of the correspondence between diminished OFC activity and level of D2 receptors found in the brains of stimulant addicts (Volkow et al, 2001b). Thus, our working hypothesis is that the mAMPH-induced loss of DA in nonmotor regions of the striatum impairs the function of the OFC, providing a neurochemical and functional basis for both the observed effects on reversal learning in animals and maladaptive decision making in humans. Whether such functional impairments would be enhanced on multiple binge dose exposures has yet to be determined. The answers to such questions can be furthered by the development of valid animal models for mAMPH effects on the brain and behavioral flexibility. Such models would, in turn, go far in developing therapeutic targets for human abusers.

\section{ACKNOWLEDGEMENTS}

This research was supported by NIDA grant (DA-12204) awarded to Dr John Marshall and the CSULA NIH MBRS program (Dr Alicia Izquierdo). We acknowledge the CSULA Office of Academic Support, the CSULA Animal Care staff, Lina Chmiel, Mike Berlin, Andrew Huggins, and Charissa Kim for their help in behavioral testing, and Dr Andrew Holmes for comments on an earlier version of the paper.

\section{DISCLOSURE}

The authors declare no conflict of interest.

\section{REFERENCES}

Bechara A, Dolan S, Denburg N, Hindes A, Anderson SW, Nathan PE (2001). Decision-making deficits, linked to a dysfunctional ventromedial prefrontal cortex, revealed in alcohol and stimulant abusers. Neuropsychologia 39: 376-389.

Belcher AM, Feinstein EM, O'Dell SJ, Marshall JF (2008). Methamphetamine influences on recognition memory: comparison of escalating and single-day dosing regimens. Neuropsychopharmacology 33: 1453-1463.

Belcher AM, O'Dell SJ, Marshall JF (2005). Impaired object recognition memory following methamphetamine, but not $\mathrm{p}$ chloroamphetamine- or d-amphetamine-induced neurotoxicity. Neuropsychopharmacology 30: 2026-2034.

Belcher AM, O'Dell SJ, Marshall JF (2006). A sensitizing regimen of methamphetamine causes impairments in a novelty preference task of object recognition. Behav Brain Res 170: 167-172. 
Belcher AM, O'Dell SJ, Marshall JF (2009). Long-term changes in dopamine-stimulated gene expression after single-day methamphetamine exposure. Synapse 63: 403-412.

Berendse HW, Galis-De Graaf Y, Groenewegen HJ (1992). Topographical organization and relationship with ventral striatal compartments of prefrontal corticostriatal projections in the rat. J Comp Neurol 316: 314-347.

Birrell JM, Brown VJ (2000). Medial frontal cortex mediates perceptual attentional set shifting in the rat. J Neurosci 20: 43204324.

Bisagno V, Ferguson D, Luine VN (2002). Short toxic methamphetamine schedule impairs object recognition task in male rats. Brain Res 940: 95-101.

Block AE, Dhanji H, Thompson-Tardif SF, Floresco SB (2007). Thalamic-prefrontal cortical-ventral striatal circuitry mediates dissociable components of strategy set shifting. Cereb Cortex 17: $1625-1636$.

Boja JW, Mitchell WM, Patel A, Kopajtic TA, Carroll FI, Lewin AH et al. (1992). High-affinity binding of [125I]RTI-55 to dopamine and serotonin transporters in rat brain. Synapse 12: 27-36.

Chung A, Lyoo IK, Kim SJ, Hwang J, Bae SC, Sung YH et al. (2007). Decreased frontal white-matter integrity in abstinent methamphetamine abusers. Int J Neuropsychopharmacol 10: 765-775.

Clarke HF, Robbins TW, Roberts AC (2008). Lesions of the medial striatum in monkeys produce perseverative impairments during reversal learning similar to those produced by lesions of the orbitofrontal cortex. J Neurosci 28: 10972-10982.

Clatworthy PL, Lewis SJG, Brichard L, Hong YT, Izquierdo D, Clark L et al. (2009). Dopamine release in dissociable striatal subregions predicts the different effects of oral methylphenidate on reversal learning and spatial working memory. J Neurosci 29: 4690-4696.

Commins DL, Seiden LS (1986). alpha-Methyltyrosine blocks methylamphetamine-induced degeneration in the rat somatosensory cortex. Brain Res 365: 15-20.

Cools R, Frank MJ, Gibbs SE, Miyakawa A, Jagust W, D’Esposito M (2009). Striatal dopamine predicts outcome-specific reversal learning and its sensitivity to dopaminergic drug administration. J Neurosci 29: 1538-1543.

Daberkow DP, Riedy MD, Kesner RP, Keefe KA (2008). Effect of methamphetamine neurotoxicity on learning-induced Arc mRNA expression in identified striatal efferent neurons. Neurotox Res 14: 307-315.

Dalley JW, Laane K, Theobald DE, Pena Y, Bruce CC, Huszar AC et al. (2007). Enduring deficits in sustained visual attention during withdrawal of intravenous methylenedioxymethamphetamine self-administration in rats: results from a comparative study with d-amphetamine and methamphetamine. Neuropsychopharmacology 32: 1195-1206.

Dalley JW, Mar AC, Economidou D, Robbins TW (2008). Neurobehavioral mechanisms of impulsivity: fronto-striatal systems and functional neurochemistry. Pharmacol Biochem Behav 90: 250-260.

Eisch AJ, Gaffney M, Weihmuller FB, O'Dell SJ, Marshall JF (1992). Striatal subregions are differentially vulnerable to the neurotoxic effects of methamphetamine. Brain Res 598: 321-326.

Eisch AJ, Marshall JF (1998). Methamphetamine neurotoxicity: dissociation of striatal dopamine terminal damage from parietal cortical cell body injury. Synapse 30: 433-445.

Everitt BJ, Belin D, Economidou D, Pelloux Y, Dalley JW, Robbins TW (2008). Review. Neural mechanisms underlying the vulnerability to develop compulsive drug-seeking habits and addiction. Philos Trans $R$ Soc Lond B Biol Sci 363: 3125-3135.

Everitt BJ, Robbins TW (2005). Neural systems of reinforcement for drug addiction: from actions to habits to compulsion. Nat Neurosci 8: 1481-1489.

Everitt BJ, Wolf ME (2002). Psychomotor stimulant addiction: a neural systems perspective. J Neurosci 22: 3312-3320.
Featherstone RE, Rizos Z, Kapur S, Fletcher PJ (2008). A sensitizing regimen of amphetamine that disrupts attentional set-shifting does not disrupt working or long-term memory. Behav Brain Res 189: 170-179.

Fletcher PJ, Tenn CC, Rizos Z, Lovic V, Kapur S (2005). Sensitization to amphetamine, but not PCP, impairs attentional set shifting: reversal by a D1 receptor agonist injected into the medial prefrontal cortex. Psychopharmacol (Berl) 183: 190-200.

Gibb JW, Stone DM, Stahl DC, Hanson GR (1987). The effects of amphetamine-like designer drugs on monoaminergic systems in rat brain. NIDA Res Monogr 76: 316-321.

Haluk DM, Floresco SB (2009). Ventral striatal dopamine modulation of different forms of behavioral flexibility. Neuropsychopharmacology 34: 2041-2052.

Han DH, Yoon SJ, Sung YH, Lee YS, Kee BS, Lyoo IK et al. (2008). A preliminary study: novelty seeking, frontal executive function, and dopamine receptor (D2) TaqI A gene polymorphism in patients with methamphetamine dependence. Compr Psychiatry 49: 387-392.

He J, Yang Y, Yu Y, Li X, Li XM (2006). The effects of chronic administration of quetiapine on the methamphetamine-induced recognition memory impairment and dopaminergic terminal deficit in rats. Behav Brain Res 172: 39-45.

Herring NR, Schaefer TL, Gudelsky GA, Vorhees CV, Williams MT (2008a). Effect of +-methamphetamine on path integration learning, novel object recognition, and neurotoxicity in rats. Psychopharmacology (Berl) 199: 637-650.

Herring NR, Schaefer TL, Tang PH, Skelton MR, Lucot JP, Gudelsky GA et al. (2008b). Comparison of time-dependent effects of $(+)$-methamphetamine or forced swim on monoamines, corticosterone, glucose, creatine, and creatinine in rats. BMC Neurosci 9: 49.

Hollerman JR, Tremblay L, Schultz W (2000). Involvement of basal ganglia and orbitofrontal cortex in goal-directed behavior. Prog Brain Res 126: 193-215.

Holmes A, Wellman CL (2008). Stress-induced prefrontal reorganization and executive dysfunction in rodents. Neurosci Biobehav Rev 33: 773-783.

Izquierdo A, Wellman CL, Holmes A (2006a). Brief uncontrollable stress causes dendritic retraction in infralimbic cortex and resistance to fear extinction in mice. J Neurosci 26: 5733-5738.

Izquierdo A, Wiedholz LM, Millstein RA, Yang RJ, Bussey TJ, Saksida LM et al. (2006b). Genetic and dopaminergic modulation of reversal learning in a touchscreen-based operant procedure for mice. Behav Brain Res 171: 181-188.

Jones B, Mishkin M (1972). Limbic lesions and the problem of stimulus-reinforcement associations. Exp Neurol 36: 362-377.

Kalechstein AD, Newton TF, Green M (2003). Methamphetamine dependence is associated with neurocognitive impairment in the initial phases of abstinence. J Neuropsychiatry Clin Neurosci 15: 215-220.

Kim SJ, Lyoo IK, Hwang J, Sung YH, Lee HY, Lee DS et al. (2005). Frontal glucose hypometabolism in abstinent methamphetamine users. Neuropsychopharmacology 30: 1383-1391.

Kolb B, Gorny G, Li Y, Samaha AN, Robinson TE (2003). Amphetamine or cocaine limits the ability of later experience to promote structural plasticity in the neocortex and nucleus accumbens. Proc Natl Acad Sci USA 100: 10523-10528.

Man MS, Clarke HF, Roberts AC (2008). The role of the orbitofrontal cortex and medial striatum in the regulation of prepotent responses to food rewards. Cereb Cortex 19: 899-906.

Marshall JF, Belcher AM, Feinstein EM, O'Dell SJ (2007). Methamphetamine-induced neural and cognitive changes in rodents. Addiction 102(Suppl 1): 61-69.

McAlonan K, Brown VJ (2003). Orbital prefrontal cortex mediates reversal learning and not attentional set shifting in the rat. Behav Brain Res 146: 97-103. 
McCann UD, Kuwabara H, Kumar A, Palermo M, Abbey R, Brasic J et al. (2008). Persistent cognitive and dopamine transporter deficits in abstinent methamphetamine users. Synapse 62: 91-100.

Monchi O, Ko JH, Strafella AP (2006). Striatal dopamine release during performance of executive functions: A [(11)C] raclopride PET study. Neuroimage 33: 907-912.

Nagai T, Takuma K, Dohniwa M, Ibi D, Mizoguchi H, Kamei H et al. (2007). Repeated methamphetamine treatment impairs spatial working memory in rats: reversal by clozapine but not haloperidol. Psychopharmacology (Berl) 194: 21-32.

Nordahl TE, Salo R, Leamon M (2003). Neuropsychological effects of chronic methamphetamine use on neurotransmitters and cognition: a review. J Neuropsychiatry Clin Neurosci 15: 317-325.

O'Dell SJ, Weihmuller FB, Marshall JF (1993). Methamphetamineinduced dopamine overflow and injury to striatal dopamine terminals: attenuation by dopamine D1 or D2 antagonists. J Neurochem 60: 1792-1799.

O'Neill M, Brown VJ (2007). The effect of striatal dopamine depletion and the adenosine A2A antagonist KW-6002 on reversal learning in rats. Neurobiol Learn Mem 88: 75-81.

Ohmori T, Koyama T, Muraki A, Yamashita I (1993). Competitive and noncompetitive $\mathrm{N}$-methyl-D-aspartate antagonists protect dopaminergic and serotonergic neurotoxicity produced by methamphetamine in various brain regions. J Neural Transm Gen Sect 92: 97-106.

Ornstein TJ, Iddon JL, Baldacchino AM, Sahakian BJ, London M, Everitt BJ et al. (2000). Profiles of cognitive dysfunction in chronic amphetamine and heroin abusers. Neuropsychopharmacology 23: 113-126.

Paxinos G, Watson C (2003). The Rat Brain Atlas in Stereotaxic Coordinates. 5th edn. Academic Press: Sydney..

Pontieri FE, Crane AM, Seiden LS, Kleven MS, Porrino LJ (1990). Metabolic mapping of the effects of intravenous methamphetamine administration in freely moving rats. Psychopharmacology (Berl) 102: 175-182.

Ragozzino ME (2007). The contribution of the medial prefrontal cortex, orbitofrontal cortex, and dorsomedial striatum to behavioral flexibility. In: Schoenbaum G, Gottfied JA, Murray EA, Ramus SJ (eds). Linking Affect to Action: Critical Contributions of the Orbitofrontal Cortex. Blackwell Publishing: Boston. pp 355-375.

Ricaurte GA, Guillery RW, Seiden LS, Schuster CR, Moore RY (1982). Dopamine nerve terminal degeneration produced by high doses of methylamphetamine in the rat brain. Brain Res 235: 93-103.

Robbins TW (2005). Chemistry of the mind: neurochemical modulation of prefrontal cortical function. J Comp Neurol 493: 140-146.

Rogers RD, Everitt BJ, Baldacchino A, Blackshaw AJ, Swainson R, Wynne K et al. (1999). Dissociable deficits in the decisionmaking cognition of chronic amphetamine abusers, opiate abusers, patients with focal damage to prefrontal cortex, and tryptophan-depleted normal volunteers: evidence for monoaminergic mechanisms. Neuropsychopharmacology 20: 322-339.

Schoenbaum G, Shaham Y (2008). The role of orbitofrontal cortex in drug addiction: a review of preclinical studies. Biol Psychiatry 63: $256-262$.
Schoenbaum G, Saddoris MP, Ramus SJ, Shaham Y, Setlow B (2004). Cocaine-experienced rats exhibit learning deficits in a task sensitive to orbitofrontal cortex lesions. Eur J Neurosci 19: 1997-2002.

Schroder N, O'Dell SJ, Marshall JF (2003). Neurotoxic methamphetamine regimen severely impairs recognition memory in rats. Synapse 49: 89-96.

Simon SL, Domier C, Carnell J, Brethen P, Rawson R, Ling W (2000). Cognitive impairment in individuals currently using methamphetamine. Am J Addict 9: 222-231.

Szumlinski KK, Haskew RE, Balogun MY, Maisonneuve IM, Glick SD (2001). Iboga compounds reverse the behavioural disinhibiting and corticosterone effects of acute methamphetamine: Implications for their antiaddictive properties. Pharmacol Biochem Behav 69: 485-491.

Takahashi Y, Roesch MR, Stalnaker TA, Schoenbaum G (2007). Cocaine exposure shifts the balance of associative encoding from ventral to dorsolateral striatum. Front Integr Neurosci 1: nihpa51247.

Tata DA, Yamamoto BK (2008). Chronic stress enhances methamphetamine-induced extracellular glutamate and excitotoxicity in the rat striatum. Synapse 62: 325-336.

Verdejo-Garcia A, Lawrence AJ, Clark L (2008). Impulsivity as a vulnerability marker for substance-use disorders: review of findings from high-risk research, problem gamblers and genetic association studies. Neurosci Biobehav Rev 32: 777-810.

Villemagne V, Yuan J, Wong DF, Dannals RF, Hatzidimitriou G, Mathews WB et al. (1998). Brain dopamine neurotoxicity in baboons treated with doses of methamphetamine comparable to those recreationally abused by humans: evidence from [11C]WIN-35,428 positron emission tomography studies and direct in vitro determinations. J Neurosci 18: 419-427.

Volkow ND, Chang L, Wang GJ, Fowler JS, Franceschi D, Sedler M et al. (2001a). Loss of dopamine transporters in methamphetamine abusers recovers with protracted abstinence. J Neurosci 21: 9414-9418.

Volkow ND, Chang L, Wang GJ, Fowler JS, Ding YS, Sedler M et al. (2001b). Low level of brain dopamine D2 receptors in methamphetamine abusers: association with metabolism in the orbitofrontal cortex. Am J Psychiatry 158: 2015-2021.

Volkow ND, Chang L, Wang GJ, Fowler JS, Leonido-Yee M, Franceschi D et al. (2001c). Association of dopamine transporter reduction with psychomotor impairment in methamphetamine abusers. Am J Psychiatry 158: 377-382.

Volkow ND, Fowler JS, Wang GJ, Swanson JM (2004). Dopamine in drug abuse and addiction: results from imaging studies and treatment implications. Mol Psychiatry 9: 557-569.

Volkow ND, Wang GJ, Telang F, Fowler JS, Logan J, Childress AR et al. (2006). Cocaine cues and dopamine in dorsal striatum: mechanism of craving in cocaine addiction. J Neurosci 26: 6583-6588.

Wilson JM, Kalasinsky KS, Levey AI, Bergeron C, Reiber G, Anthony RM et al. (1996). Striatal dopamine nerve terminal markers in human, chronic methamphetamine users. Nat Med 2: 699-703. 